\title{
TERRITORIALIDADES, CONHECIMENTOS, TRANSFORMAÇÕES E CONFLITOS EM UM ESTUDO DA PESCA ARTESANAL EM LAGUNA GARZÓN, URUGUAI
}

\author{
TERRITORIALITIES, KNOWLEDGE, \\ TRANSFORMATIONS AND CONFLICTS \\ IN A STUDY OF ARTISANAL FISHERY \\ IN LAGUNA GARZÓN, URUGUAY
}

TERRITORIALIDADES,

CONOCIMIENTOS, TRANSFORMACIONES Y CONFLICTOS EN UN ESTUDIO DE LA PESCA ARTESANAL EN LA LAGUNA GARZÓN, URUGUAY

\section{Dra. Leticia D'Ambrosio Camarero}

treboles@gmail.com (CURE, UDELAR) Profa.Adjunta Centro Universitario Regional Este/Universidad de la República. Dra. en Antropología Social (IDAES-UNSAM), Mag. en Ciencias Sociales (FLACSO Argentina), Lic. en Ciencias Antropológicas (FHCE-UDELAR). Directora del Centro de Investigaciones del Patrimonio Costero (CIPAC-CURE-UDELAR).

Orcid: https://orcid.org/0000-0001-8181-9955

\section{Lic. Inti Clavijo}

inticlavijo@gmail.com (CURE, UDELAR) Lic.en Ciencias Antropológicas opción Social y Cultural (FHCEUDELAR). Integrante del Centro de Investigaciones del Patrimonio Costero (CIPAC). Orcid: https://orcid.org/0000-0003-3870-660X

M.Sc. Viviana Cuberos Betancourt

vivicuberos@gmail.com (CURE,UDELAR) M. Sc.mención Antropología (Instituto Venezolano de

Investigaciones Cientificas), Antropóloga (Universidad Central de Venezuela), Integrante del Centro de Investigaciones del Patrimonio Costero (CIPAC).

Orcid: https://orcid.org/0000-0003-3038-8352

\section{RESUMO}

O presente artigo apresenta alguns aspetos do resultado de uma pesquisa etnográfica sobre as territorialidades, temporalidades e os conhecimentos encológicos pesqueiros, as transformações e os diversos conflitos observados em torno à pescaria artesanal da Laguna Garzón em Uruguai. A partir do trabalho de campo tem sido reveladas diferentes formas de categorização e significação da lagoa, o oceano, a barra, os peixes e seu entorno, que dão conta do conhecimento gerado nas práticas da localidade, que no nosso critério resultam interessantes de se cristalizar neste artigo. Através das diferentes interlocuções com os pescadores da zona, revela-se uma rede discursiva que expõe di- 
ferentes elementos que caracterizam uma pescaria que possui algumas particularidades no Leste do território uruguaio. Esta articula diferentes espaços entre o político e o jurídico assim como os conhecimentos ecológicos locais e os saberes tradicionais dos povoadores em relação à dinâmica médio-ambiental da área. A territorialidade nativa e as formas de geo-referenciamento na lagoa vinculam-se com a mobilidade e as características das espécies capturadas assim como à conexão da água doce e salgada com o espaço oceânico. O entorno está demarcado por um grande dinamismo onde interatuam humanos e não-humanos. No percurso da pesquisa o trabalho etnográfico tem permitido a aproximação com as diferentes formas de construção do entorno da lagoa que conjuga-se na cotidianidade da localidade, por enquanto em outros momentos desencontra-se e faz emergir diversos conflitos que ainda hoje continuam sem ter sido resolvidos.

Palavras Chave: Pescaria artesanal. Territorialidade. Cosmografia.

\begin{abstract}
This article presents interesting aspects of the territorialities, knowledge, transformations and conflicts that have been observed around the artisanal fishery of Laguna Garzón in Uruguay. As a product of the ethnographic research different forms of categorization and significance of the lagoon, the ocean, the bar and its environment have been revealed. These elements account for the knowledge generated in the practices of the locality, which in our opinion are interesting to crystallize in this article. Through different conversations with fishermen in the area and other social actors present there, such as tour operators and state officials, a discursive plot is revealed, which exposes different elements that characterize a fishery that has some peculiarities in the East of the Uruguayan territory. This plot articulates different aspects of the political and legal sphere as well as the local ecological knowledge of the inhabitants in relation to the environmental dynamics of the area. The native territoriality and the georeferenced forms in the lagoon are linked to the mobility and characteristics of the species captured as well as to the connection of fresh or brackish water with the oceanic space. The environment is marked by a great dynamism in which humans and non-humans interact. In the course of the investigation, the ethnographic work made possible to approach different ways of constructing the environment of the lagoon. As well as this is conjugated in the daily life of the locality, in other moment it is mismatched and emerges several conflicts that still continue without resolution.
\end{abstract}

Keywords: Artisanal fishery. Territoriality. Cosmography.

\title{
RESUMÉN
}

El presente artículo es el resultado de una investigación etnográfica sobre las territorialidades, temporalidades, los conocimientos ecológicos pesqueros, las transformaciones y los diversos conflictos que se observan en torno a la pesquería artesanal de Laguna Garzón en Uruguay. A partir del trabajo de campo se han ido revelando diferentes formas de categorización y significación de la laguna, el océano, la barra, los peces y su entorno, que dan cuenta del conocimiento generado en las prácticas de la localidad, que a nuestro criterio resultan interesantes de cristalizar en este artículo. A través de diferentes interlocuciones con los pescadores de la zona se revela una trama discursiva que expone diferentes elementos que caracterizan una pesquería que posee algunas peculiaridades en el Este del territorio uruguayo. Esta articula diferentes espacios entre lo político y jurídico así como los conocimientos ecológicos locales y los saberes tradicionales de los pobladores en relación a la dinámica medioambiental del área. La territorialidad nativa y las formas de georeferenciación en la laguna se vinculan con la movilidad y características de las especies capturadas así como a la conexión del agua dulce o salobre con el espacio oceánico. El entorno está marcado por un gran dinamismo en el que interactúan humanos y no-humanos. En el transcurso de la investigación la labor etnográfica ha permitido tomar cercanía con diferentes formas de construir el entorno de la laguna que se conjuga en la cotidianidad de la localidad, mientras que en 
otros momentos se desencuentra y hace emerger diversos conflictos que aún continúan sin resolución.

Palabras clave: Pesca artesanal. Territorialidades. Cosmografías.

\section{INTRODUCCIÓN}

Este trabajo surge como parte del proyecto "Pesquerías artesanales en la costa Este de Uruguay: Aportes para su investigación y gestión" en el que, con un enfoque sociocultural e histórico, abordamos las pesquerías artesanales en la costa Este de Uruguay. Específicamente en este artículo analizaremos las territorialidades y cosmografías que existen en Laguna Garzón, las formas de geo-referenciación y finalmente buscamos comprender los conflictos presentados por las transformaciones en el área, desde la perspectiva de los pescadores artesanales. Para ello, utilizamos un enfoque etnográfico en el que la observación participante y las entrevistas en profundidad fueron nuestras principales herramientas de investigación.

\section{LAGUNA GARZÓN: CARACTERIZACIÓN GENERAL Y PARTICULARIDADES}

La localidad de Laguna Garzón se ubica en el límite departamental entre Maldonado y Rocha. La laguna, así como el espacio terrestre circundante, se insertaron en una Área Protegida del SNAP (Sistema Nacional de Áreas Protegidas) desde el año 2014. Cabe mencionar que, recién en el año 2019, se inicia un proceso para la definición de un Plan de Manejo del Área.

En los alrededores de la laguna se pueden encontrar alrededor de una decena de familias, algunas de las cuales desarrollan la pesca artesanal de forma regular. La mayoría de ellos vive en la costa del espejo del agua y practican la pesca artesanal en la laguna, aunque algunos se movilizan hacia otras lagunas de la región lacustre del Este uruguayo, siguiendo la zafra de las diferentes especies. Y otros pobladores se dedican a otro tipo de actividades como la comercialización de otros bienes naturales como leña. Los principales destinos de esta movilidad suelen ser Laguna de José Ignacio, la más cercana a Garzón, Laguna de Rocha, que está un poco más lejana, entre los relatos también aparece la captura del camarón como otra zafra a la que solían concurrir en la Barra de Valizas.

Si bien esta movilidad aparece con frecuencia en los relatos de los interlocutores de esta investigación, para gran parte de los pescadores asentados en la zona, esta movilidad es cosa del pasado, muchos ya dejaron de moverse y optaron por quedarse asentados en La Laguna de Garzón (principalmente por su avanzada edad), pescando solamente allí, aunque las capturas mermen considerablemente y las ganancias no permitan un rédito mayor al necesario para la subsistencia. Muchos de los residentes sobrepasan los setenta años y llevan ya al menos treinta años residiendo allí. La edad avanzada influye también en la elección de trabajar en la laguna y no en el mar. Al respecto, es interesante la categorización que se hace de ambos entornos, por un lado, la laguna se percibe como un agua tranquila, dócil, fácil y suave de recorrer y trabajar, en contrapartida a esta descripción, el mar está asociado a la fuerza y la exigencia física, a una destreza y esfuerzo corporal al que muchos de ellos prefieren ya no exponerse. Surge también, en el mar, la preocupación por el riesgo. Este último aspecto ha sido analizado por diversos autores dentro de la antropología 
marítima (MALDONADO, 1994; ADOMILLI, 2007; COLAÇO, 2015), quienes han señalado que es un componente presente en aquellos que trabajan o realizan actividades en el mar. "La interacción con el entorno marítimo costero presenta el desafío del encuentro con lo desconocido que implica una aventura cotidiana, y de lidiar con lo inesperado, con características más dinámicas e imprevisibles que las atribuidas a la tierra" (MALDONADO, 1994). En el caso de las pesquerías de este estudio, la laguna pareciera en este aspecto, acercarse más a las características atribuidas a la tierra. Mientras que en el mar, como lo señala Maldonado para su estudio sobre los pescadores artesanales:

“esas prácticas y modos de vida se construyen en relación a un medio tanto física como socialmente inestable e imprevisible. El mar, espacio de vida de los pescadores marítimos, es marcado por la fluidez de las aguas y de sus recursos, por la inestabilidad continua provocada por factores meteorológicos y oceanográficos..." (GEISTDOERFER apud MALDONADO 1994,158).

En el estudio que presentamos aquí, los pescadores artesanales se han dedicado a la práctica de la pesca en la laguna con las características que veremos a continuación.

Figura 1 - Vista de la costa de la laguna a las casas de los pescadores

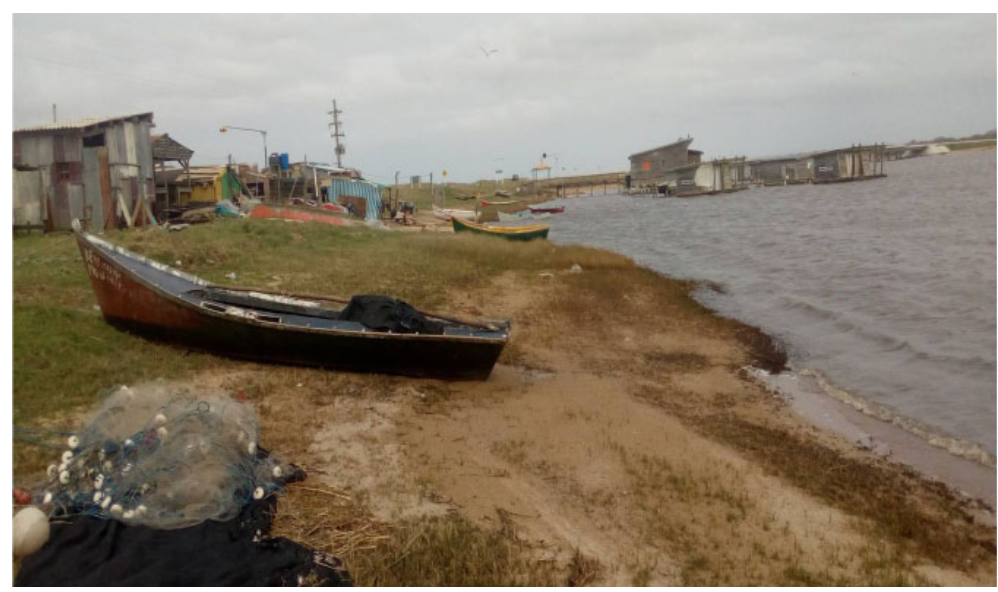

Fuente: Inti Clavijo.

\section{PESCA, CONOCIMIENTOS Y GEO- REFERENCIACIÓN}

La laguna Garzón tiene en el cuerpo de agua una extensión de dos mil hectáreas de acuerdo a los gestores del área. Los pescadores que llevan más tiempo allí la conocen en detalle y profundidad, e incluso han llegado a recorrer y a pescar en los arroyos afluentes, los cuales entienden como una continuidad de la Laguna.

Podemos decir que, en torno a la misma, han generado un conocimiento ecológico desarrollado en las prácticas de la localidad. Lo que INGOLD y KURTTILA (2000) entienden como aquel conocimiento tradicional generado en las prácticas de localidad por oposición al modelo que llaman genealógico. Dicho conocimiento y sus prácticas asociadas, orienta y sustenta el funcionamiento de sistemas de manejo comunitario. Siendo el mismo un conocimiento empírico y práctico, producto de un proceso acumulativo y dinámico de experiencias prácticas y de adaptación al cambio. Es local, holístico y portador de 
una cosmovisión que integra aspectos físicos y espirituales. Consideramos que este concepto es útil para abordar los datos empíricos relevados en el presente caso de estudio, pues permite acercarnos a una idea dinámica del conocimiento y al mismo tiempo a un conocimiento y habilidades generadas en las prácticas de la localidad. Como veremos más adelante hay modalidades de pesca y de geo-referenciación que forman parte de las particularidades de las pesquerías en esta laguna en particular.

El cuerpo de agua tiene poca profundidad, según los pescadores, no supera los dos metros en su momento más crecido. Esta escasa profundidad y su acotada superficie para la pesca hacen que no sean empleados medios de geo-localización digitales o sondas para la localización de los peces, de manera que, no son utilizados los GPS ni las Eco-sondas generalizadas en la pesca marítima artesanal de la región (D'AMBROSIO et al 2010, D'AMBROSIO, 2017b). Al respecto, uno de los pescadores con quien conversamos en Laguna Garzón, pero que reside y pesca habitualmente en Laguna de Rocha, relataba:

Er1: ¿Cómo hacen, usan GPS o algo?

Et: Puedo tener un GPS, pero soy bruto, no tengo conocimiento, fui a poca...y no me gusta a mí, lo que me gusta a mí es la tranquilidad, no soy hombre de ciudad, ni de andar chupando.

Er1: ¿Y cómo se orienta cuándo sale así y hay mucha niebla? ¿cómo hace?

Et: Una brisita.

Er1: ¿Por la brisa se orienta?

Et: Viento abajo viento arriba, viento de costado a veces.

Er1: ¿Y cuándo hay temporal no sale?

Et: No no.

Er2: ¿Qué vendría a ser el viento abajo? ¿viento abajo qué es?

Et: Por ejemplo si está al sureste usted va pa allá.

Er2: ¿Cuál es el mejor viento para ir a pescar?

Et: Y del este norte viento de tierra como le dicen en el mar.

Er1: ¿Viento de tierra le dicen?

Et: Viento de tierra.

De modo que todas estas innovaciones que en la pesca marina han tenido un efecto muy significativo para poder localizar los cardúmenes y marcar los sitios de pesca, no han sido efectivos o necesarios en la laguna.

Por otro lado, el conocimiento de la laguna y sus décadas de práctica han generado un conocimiento acumulado de geo-referenciación local, que se ha producido por los mismos pescadores y se ha perpetuado hasta el día de hoy. Este sistema comprende la delimitación de ciertas "cacholetas" o "piedras" de referencia. 
Figura 2 - Laguna Garzón con sus afluentes

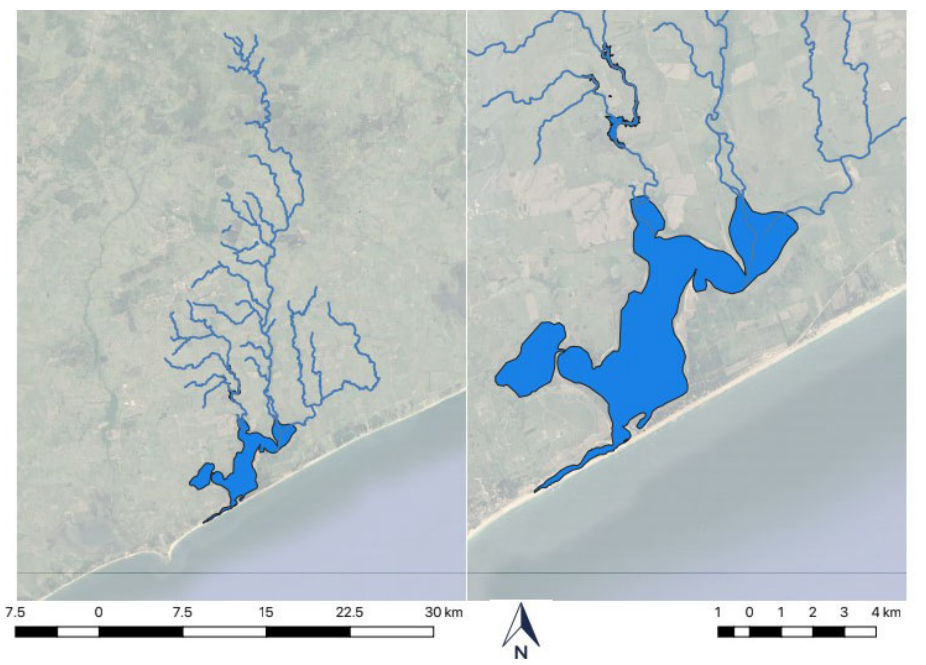

Fuente: Eduardo Keldjian.

Figura 3 - Foto satelital de Laguna Garzón (2018)

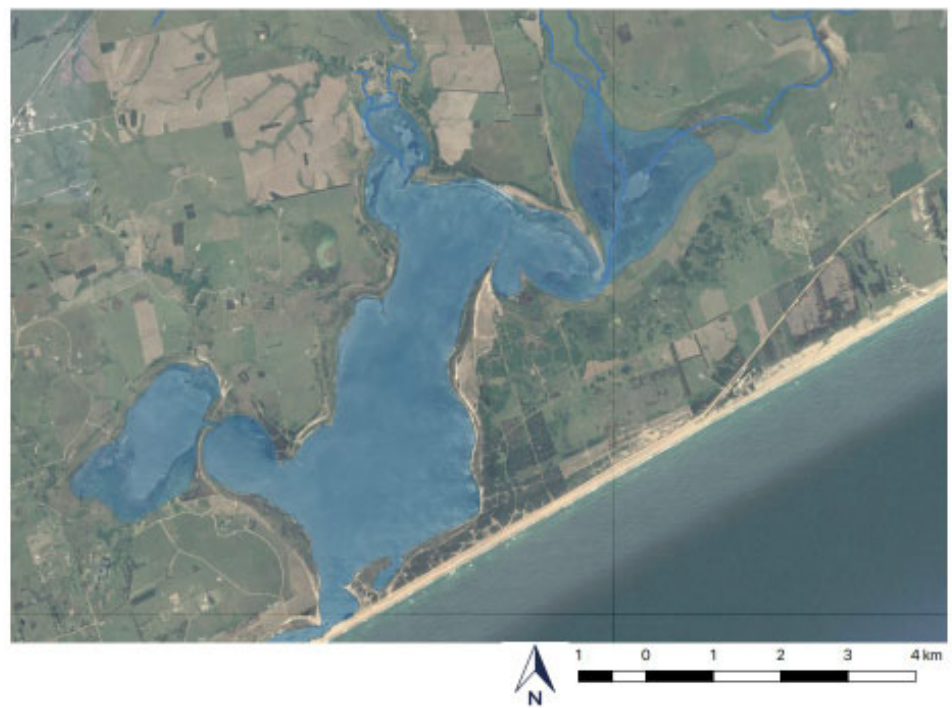

Fuente: Eduardo Keldjian.

Así, los nombres como la Cacholeta de las Hormigas, la de la Piedra Alta, la de la Piedra del Brasilero, la Cacholeta del País, la de las Aguas Blancas, corresponden, muchas veces, a lugares de referencia, como las piedras, o bien a características del agua, como la de Aguas Blancas y también de algunas chacras de la costa como la del Brasileño o País. "Vinculado con esto, MALDONADO (1994) observa que este aspecto está relacionado con una de las dimensiones de la camaradería, que refiere a la experiencia compartida en la ocupación común, a representaciones simbólicas también compartidas por el grupo como identidad común" D’AMBROSIO (2017a,150). Estas denominaciones provienen de más tiempo que el que todos dicen llevar ahí, son de alguna forma una herencia en forma de "tradición inventada" HOBSBAWM y RANGER (2002). Según estos autores:

"Se emplea el término tradición, inventada en sentido amplio, pero no impreciso. Incluye por igual tradiciones efectivamente inventadas, elaboradas y formalmente establecidas, y aquellas que emergen en forma no tan fácilmente rastreable dentro de un período breve e identificable 
cronológicamente - en cuestión de unos pocos años, quizás - y que se fijan con gran rapidez" (HOBSBAWM y RANGER 2002: 7).

Al mismo tiempo este conocimiento se comparte entre los pescadores considerados como iguales a diferencia de los "otros" pescadores que vienen zafral o esporádicamente, y a quienes se los llama con la categoría nativa de "turistas". La misma surge como alteridad de los pescadores artesanales residentes desde hace varios décadas o generaciones en el área circundante a la laguna.

Figura 4 - Vista a la lengüeta de la Laguna y barra arenosa

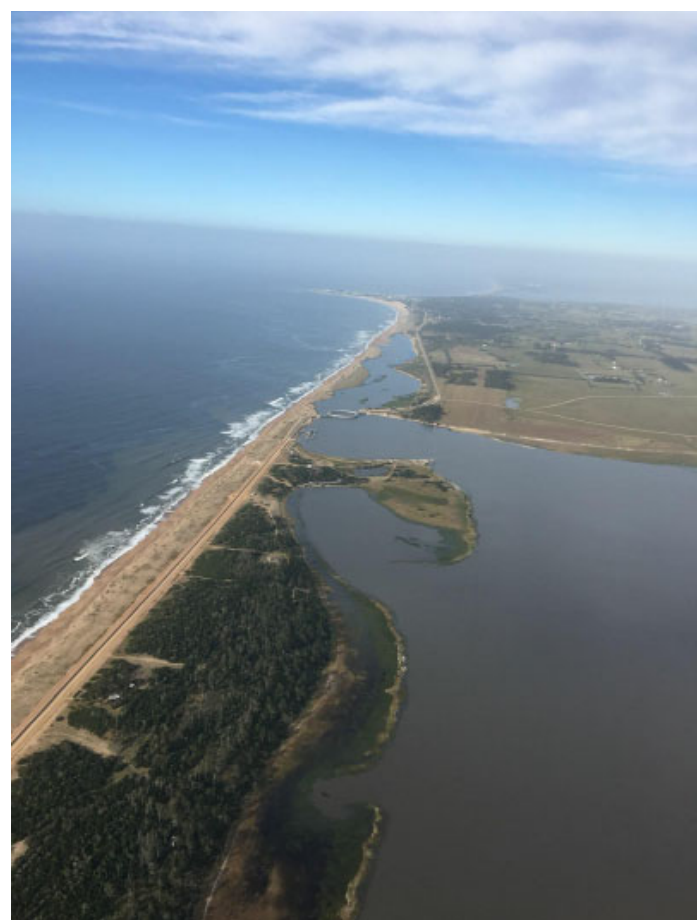

Foto: Carlos Amorin (Pescador Artesanal)

Los puntos de referenciación sirven para comunicar y recordar los datos en relación a la práctica pesquera, pero que para navegar es difícil perderse teniendo en cuenta su tamaño. Al respecto, cuando uno de los pescadores cala en cierto lugar, usa estos sitios de referencia para poder comunicar a otros, o bien para no comunicar, ya que el "secreto de pesca" es también muy común. El mismo refiere a esa práctica de guardar para sí mismos la información de los sitios donde han encontrado peces para utilizarlo de referencia en la siguiente salida. Al respecto, el secreto ha sido uno de los temas clásicos de la antropología de la pesca en los que se señala que la presencia del secreto y la mentira son una estrategia de asegurar el control y la propiedad sobre los bienes. En este sentido GALVAN y FERNÁNDEZ (1996) observan que en tanto que:

"el grupo no controla directamente los recursos [...], el acceso a los recursos se intenta restringir mediante el control sobre el conocimiento de los lugares de pesca, que se transmite por lazos de parentesco u otros. Su carácter secreto limita el flujo de datos no solo a los pescadores de fuera de la comunidad [...] Por tanto, la distribución territorial de las poblaciones y sus áreas de actividad pesquera no se realiza mediante fronteras espaciales, dado que el territorio está abierto, sino gracias a fronteras sociales de grupos y unidades domésticas, y a la distribución desigual del conocimiento sobre la geografía y los recursos" (GALVAN Y FERNÁNDEZ 1996). 
En relación a los lugares de pesca, otro de los pescadores dice que no se comparten mucho los "piques" donde hay peces, sino que suelen guardarlos para su próxima captura como decíamos anteriormente, aplicando el secreto. Aunque resultaría sencillo reconocer de dónde viene un pescador que llegó a la costa y trae sus redes cargadas:

Er1: ¿Y por ejemplo, cuándo ven que hay mucho pescado en una zona le avisan a otros, a los amigos, y eso, a otros pescadores o no?

Et1: No no, mayormente no digo, mayormente no digo, ta' cada uno está viendo más o menos y te ve andar en la pesca digo y ya cuando ves pasar.

Et2: Lo dejo pal otro día yo, jajaja.

Er1: No dice nada.

Et2: No digo nada.

Et1: Si traes pescado se dan cuenta, digo es fácil.

Er2: De donde venís.

Et1: Esto es tan chiquito.

Er1: Claro se ven cuando vienen ya con la...

Et1: Claro, él que quiere más o menos está en la pega va más o menos ta' fulano agarró en tal lado que siempre están mirando uno a otro el pescador, donde agarró donde no... o sea que trae y que no trae.

La cercanía de las casas con la costa de la laguna y la permanencia de los pescadores y sus familias, en sus casas, durante gran parte del día hace que tengan conocimiento de la situación de pesca del día o de "la suerte" que han tenido los otros pescadores. Considerando la incertidumbre que se produce diariamente en la actividad de la pesca artesanal, ya que, la disponibilidad de "pescado" varía de un día al otro, esta transmisión de conocimientos se vuelve fundamental:

Et1: La pesca es como la quiniela, a veces sales y no agarrás nada, a veces llenas un bote.

Et2: Hoy nadie agarró, lenguado nadie agarró hoy, yo no agarré (...)

Er1: ¿Cómo sabe? ¿Por qué ve que no llegan con... que no agarraron nada? ¿Cómo sabe que los otros no agarraron tampoco?

Et1: Y porque se ve, como estamos todos acá claro.

Et2: Estamos todos acá, todos nos comunicamos ¿cuánto agarraste, cuánto no agarraste?

Er2: ¿Y vos salís siempre más o menos al mismo lugar o tenés algún?

Et2: No, no, cambiamos.

Er2: ¿Vas viendo?

Et2: Vamos viendo por eso nos comunicamos.

Er3: ¿Y con los otros se coordinan?

Et2: Seguro.

Et1: El pescado camina, no está siempre en el mismo lugar.

Et2: No calamos donde calan ellos.

Et1: Sí cada uno se respeta.

Et2: Nos respetamos, si estás calando ahí yo no voy a calar ahí, calo unas cuadras más y ellos lo mismo. 
Er3: El otro día me decías que más o menos unas ¿cuántas cuadras? ¿unas tres u ocho cuadras?

Et2: Cuatro cuadras más o menos, uno cala enfrente otro cala pal costado. ¡Mi negra pasa da la vuelta!, da la vuelta! esa es mi nieta trabaja en...

Er3: En el hotel ahí.

Esa suerte de panóptico vecinal impide que pueda aplicarse con mucha eficacia el secreto. El mantener el secreto, en este caso se ve dificultado; como señala SIMMEL (1927), al encontrarse "en un círculo reducido, de relaciones estrechas, la formación y mantenimiento de secretos se hallará dificultada por la razón técnica de que los miembros están demasiado cerca unos de otros, y porque la frecuencia e intimidad de los contactos provoca en demasía las tentaciones de revelación" (SIMMEL, 1927).

En este mismo sentido, es difícil ocultar en la laguna los lugares de pesca, ya que, como veremos a continuación los materiales suelen permanecer muchas horas en el agua. Asimismo, el uso de boyas con banderines de tela y colores identificatorios, hace que unos y otros puedan saber a quién pertenecen los materiales de pesca que se encuentran colocados al salir a la laguna. Dado que son pocos los pescadores que están de forma permanente, es sencillo reconocer a quién pertenecen los materiales.

Figura 5 - Pescadores desenmallando en Laguna Garzón (2014)

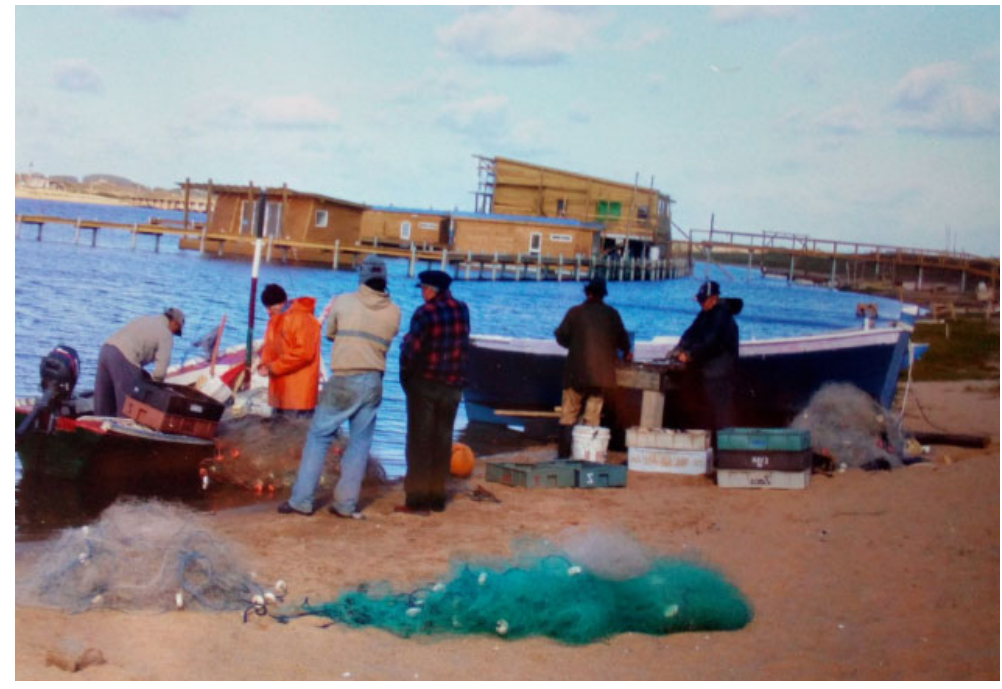

Fuente: Cortesía Pescador Artesanal

\section{CAPTURA: ESPECIES Y TEMPORALIDADES}

Generalmente, las artes de pesca permanecen toda la mañana o tarde colocados para capturar peces. Así, la práctica suele consistir en colocar las redes o trasmallos temprano en la mañana, frecuentemente antes de la salida del sol, e ir a recogerlas por la tarde, donde algunos de los pescadores ya aprovechan el viaje para colocar otras redes, aunque esto dependerá de la cantidad de "pescado" y de la voluntad o necesidad de capturar más. Después de la captura, se procede al "desenmallado", es decir, sacar los pescados de las redes, en algunos casos se realiza en el mismo lugar de pesca, dejando el "pescado" dentro del barco, mientras que en otras ocasiones, cuando el agua está un poco más agitada, se desenmalla luego de llegar a la costa. Al respecto, dos pescadores observan: 


\section{Er1: ¿Sacándolo tarda más o eso se saca rápido?}

Et1: No porque traemos para acá pa' costa, levantamos con todo, a veces, estando calmo porque hay poco pescado se desenmalla allá.

Et2: Que no se mueve el barco digamos.

Et1: Sí, habiendo viento siempre se trae a costa.

Luego de recogidas las redes, inmediatamente se procede a la limpieza del pescado que se realiza en la costa de la laguna sobre alguna mesa. Es muy frecuente que se acerquen una importante cantidad de aves aprovechando los desperdicios de la limpieza para su alimentación, por dicha razón es sencillo divisar cuando alguno de los pescadores está realizando dicha tarea, ya que una nube de aves revolotea sobre él. Luego de limpio y fileteado el pescado, es guardado en el freezer, cada pescador tiene el suyo propio. Una de las pescadoras nos decía que había un secreto importante para mantener la frescura del pescado $\mathrm{y}$ era que el freezer era prendido unas horas antes de que el pescado llegara y cuando esté era guardado, se apagaba el freezer, conservando el frío, pero sin llegar a congelar los filés, de manera que se mantiene fresco y no modifica la estructura y textura de la carne como sucede en el proceso de congelamiento. En este sentido, observamos que, por lo general hay una división por género del trabajo. Las mujeres pocas veces salen a pescar y cuando lo hacen, van junto con sus maridos. En cambio, se dedican mayormente a las tareas que corresponden a la fase de procesamiento de las especies. Si bien estas tareas también son desarrolladas por hombres.

La vía de comercialización para varios de los pescadores suele ser por la mediación de compradores que van a buscarlo directamente a sus casas, pues, por lo que nos contaron, en uno de los casos este pescado es comercializado en una pescadería de Rocha. Luego de capturado y fileteado el pescado está pronto para vender, por lo que los pescadores se comunican telefónicamente con su comprador y lo mantienen en el freezer hasta que este viene a buscarlo. Ocasionalmente, se vende el pescado al público directamente, esto sucede cuando algunos clientes vienen o llaman para preguntar si hay pescado. Sin embargo, una de las familias hace venta al público directo de manera permanente. Esta misma familia, con frecuencia, sale a pescar al mar, es también la familia más cercana a la costa marítima de las que viven en la zona. Existen algunos pescadores que venden también de forma itinerante en Maldonado y Piriápolis, pero no venden solamente sus capturas en la laguna sino que compran a otros también, dado que los volúmenes de la laguna no son muy significativos y es necesario que el viaje rinda para cubrir los costos del traslado.

Es importante considerar también que, la disponibilidad de peces suele variar a lo largo del año, algunas especies son más zafrales y se capturan solamente en determinadas épocas. Durante el trabajo de campo se pudieron relevar algunas como el pejerrey [Odontesthes bonariensis], la corvina [Micropogonias furnieri] y el lenguado [Solea solea] principalmente. Como veremos, no existe una sola forma o procedimiento para realizar la captura. En este caso, podemos ver como se alternan las especies que cada pescador captura y los diferentes horarios en los que se puede desarrollar el proceso de extracción. Algunos colocan las redes de pejerrey en la mañana para cambiarlas por las redes de lenguado en la noche, mientras que otros colocan las redes de pejerrey por la tarde para recogerlas en la mañana, así se describe en el siguiente extracto del relato de un interlocutor:

Er1: ¿Y cuáles pone normalmente?

Et1: Malla de pejerrey.

Er1: De pejerrey sobre todo ¿y lo deja un día? 
Et1: No, no, la noche.

Er1: La noche y después lo busca.

Et1: Al otro día temprano se va a buscar.

Er1: Por ejemplo ¿hoy va a buscar no?

Et1: No, poquito, una caja más o menos de pejerrey entero son diez, doce kilos que tiene.

Er1: ¿Y eso nos decían la otra vez que tienen que prepararlo, no? no lo venden entero, no lo compran entero.

Et1: No.

La temporalidad y modalidad de pesca también se ve modificada por cambios en la temperatura del agua, puesto que, al aumentar la temperatura del agua, lo que ocurre por lo general en los meses de verano, no es posible dejar las redes sumergidas por mucho tiempo, porque los pescados se conservan menos tiempo en estado apto para el consumo humano, debido al calor.

La captura del lenguado suele ser más lenta que la del pejerrey, de acuerdo al relato del interlocutor siguiente:

$\operatorname{Erl}: ¿ Y$ a vos por qué te gusta más el...?

Et1: Y ta' queda feo decirlo pero quizás sea un trabajo más de haragán que...

Er1: Aja ¿por qué cuál es la diferencia con el pejerrey?

Et1: La diferencia es que tú el lenguado de repente las ponés las redes y de repente viste que vas un par de horas o tres horas y las dejás en el agua después claro no hay duda que tenés que ir a hacer dos o tres horas o cuatro más viste y depende como estén y ahí viste o sea de un día pal otro de repente, entonces lo hacés más aliviado.

Entonces, podríamos decir que, algunos pescadores prefieren la captura del lenguado porque requiere menos constancia para salir en el barco. Pese a eso, es más frecuente la captura del pejerrey por no requerir mucho trabajo en el fileteado como sí lo requiere el lenguado, ya que para el pejerrey suele aplicarse la técnica conocida como "la palomita", que deja la pulpa de ambos lados del pescado unida enteramente a la cola de forma sencilla:

Er1: Ustedes empezaron a hacerlo así en filé, ¿sabe por qué?

Et1: Eso lo vendemos, porque ellos lo vendían en Montevideo.

Et2: Claro, porque no, eso fue una argentina que vino con la palomita de pejerrey acá...

Er1: Ahá.

Et1: (...) (Angelita) Fue la que arrancó. Fue la que empezó a hacer así el filé de pejerrey.

Er2: Ah, mirá.

Et1: Angelita, era capataz, argentina era, sabía mucho. Fue la primera que empezó a hacer. Porque nosotros cuando queríamos comer un filé de pejerrey hacíamos un filé de cada lado y estaba. Se destrozaba todo. Ella me enseñó a hacer la palomita.

Er2: Ella te enseñó.

Et1: Me enseñó y ahí sí. Aprendió todo el mundo.

Er2: La palomita, le sacas el espinazo, la cabeza y queda... 
Et1: Seguro, después te voy a mostrar que tengo ahí. Es distinto sacar un filecito así... De pejerrey de cada lado, a hacer una palomita, sacarle el espinazo y queda todo entera.

Como mencionábamos anteriormente, por lo general el fileteado es una de las tareas que realizan mayormente las mujeres, aunque no en exclusividad.

Las diferentes especies mencionadas anteriormente, marcan también una temporalidad anual variando su abundancia o escasez, lo cual hace fluctuar también la práctica de la pesca. Pese a ello, algunas especies como el pejerrey son resaltadas por los pescadores porque parece estar disponible durante todo el año:

$$
\begin{aligned}
& \text { Er: Para él y las otras... ¿por ejemplo el pejerrey cómo es? } \\
& \text { Et: El pejerrey todo el año. } \\
& \text { Er: Todo el año. }
\end{aligned}
$$

Et: El pejerrey todo el año, a veces que pecha más en invierno pero ta' lo pescas igual porque sino lo agarras con la redes en el agua puestas fija, le tirás así lo sacas entiendes, entonces lo tenés, habiendo pescado lo tienes todo el año.

Et: Tratás de más bien pescar, aunque para el lenguado no tenés vuelta supone, o sea él es mucho con el pejerrey yo más bien le doy al lenguado, o sea soy mucho pa pescar el lenguado y el lenguado sí, el lenguado es una cosa que tenés que darle reposo, no tenés vuelta, porque es una cosa lenta también, entonces estás obligado a dejarlo de hoy para mañana o dos días, depende, si es frío le dejás las redes dos días igual en el agua.

\section{Er: ¿Y el pejerrey?}

Et: El pejerrey estás obligado a echarlo y sacarlo, buscarlo, sacarlo de las redes y estás obligado a limpiarlo, a prepararlo enseguida porque el pejerrey es una cosa que se estropea rápido.

Como vemos, en algunos casos, la estacionalidad puede no ser tan determinante, allí la disponibilidad de la especie se ve sujeta a otras variables naturales y socio-ambientales del entorno de la laguna, de acuerdo a las observaciones realizadas por los interlocutores.

Figura 6 - Pescado fileteado: Pejerrey a la palomita a la izquierda, Lenguado a la derecha

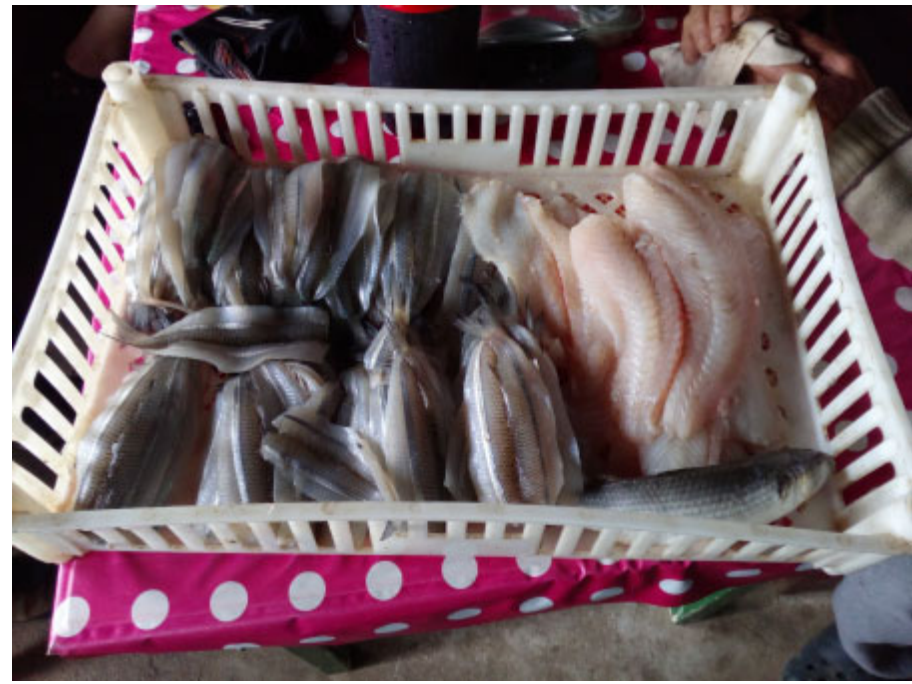

Fuente: Inti Clavijo. 


\section{DINÁMICAS SOCIO-AMBIENTALES DE LA LAGUNA}

Una parte importante del conocimiento ecológico generado en las prácticas de la localidad, refiere a las dinámicas de la laguna en la que intervienen humanos y no-humanos. De acuerdo con lo que observan los interlocutores, existe una cantidad de factores que influyen en estas dinámicas, los vientos, la fase lunar, así como también, las corrientes de agua dulce y salobre y la cantidad de cada una de estas presentes en el cuerpo de la laguna. Estas cantidades varían mucho, ya que, esta se presenta como un espacio intermedio entre el mar y los arroyos afluentes, y parece no tener agua propia en la percepción de los pescadores. Esta visión hace que surjan también especulaciones y teorías de qué cantidades de agua dulce y salada debe tener el cuerpo de la laguna para que la cantidad de peces sea más abundante. Al respecto, uno de los pescadores nos decía que debía tener al menos la mitad de agua salada para estar en su mejor condición para la pesca. Las dinámicas del agua, a través de la barra de la laguna, por estar conectada por ella con el mar, hace que influya, ya que del mar provienen los peces, algunos de los cuales desovan en la laguna, como es el caso de la "corvina cantora" [Micropogonias furnieri].

La conexión del agua dulce con el agua salada en las lagunas es un tema que ha surgido en muchas investigaciones de pesquerías artesanales. En este sentido, observamos similitudes en las formas de describir estas dinámicas entre los pescadores de Laguna Garzón y los del caso estudiado por MOURA y DIEGUES (2009) en el Saco do Arraial en la Laguna dos Patos al sur de Brasil. Por ejemplo, en terminologías que identifican las acciones que el agua realiza como cuando "as aguas se péixam", es decir "cuando el agua salada pecha al agua dulce" como nos decía un pescador de Laguna Garzón. Del mismo modo, ambos grupos de pescadores comprenden a la barra como la conexión de la laguna con el océano (MOURA y DIEGUES 2009,364); y que por tanto, está es determinante en todo lo que ocurre en relación a la pesca. En la misma dirección, el estudio de DA SILVA MELLO y VOGEL (2017) en Maricá- RJ, da cuenta del significado del momento de apertura de la barra y el pasaje del agua salada, para los pescadores, quienes lo describen como "el matrimonio entre las aguas del mar con las aguas de la laguna adquieren una relevancia especial" (DA SILVA MELLO; VOGEL, 2017,71). Y los antropólogos observan, a partir del relevamiento del conocimiento ecológico de los pescadores en Maricá- RJ que, "la laguna que resulta de ese proceso dependerá no solamente de la relación con las aguas de tierra, para la reproducción de su variada y múltiple riqueza biológica sino que además de los caminos de la vida que vienen del mar" (DA SILVA MELLO, VOGEL 2017,84). Los autores mencionados ponen de relieve el significado que los pobladores, muchos de ellos pescadores artesanales, daban a la apertura artificial de la barra. Acción que considerada como un rito colectivo, en el que además de trabajo se compartían canciones y alimentos. Este proceso es descripto por los nativos como "la fecundación del mar a la laguna" (DA SILVA MELLO y VOGEL, 2017). 
Figura 7 - Puesto de venta de familia pescadora con Barra Arenosa de Playa en el fondo (2017)

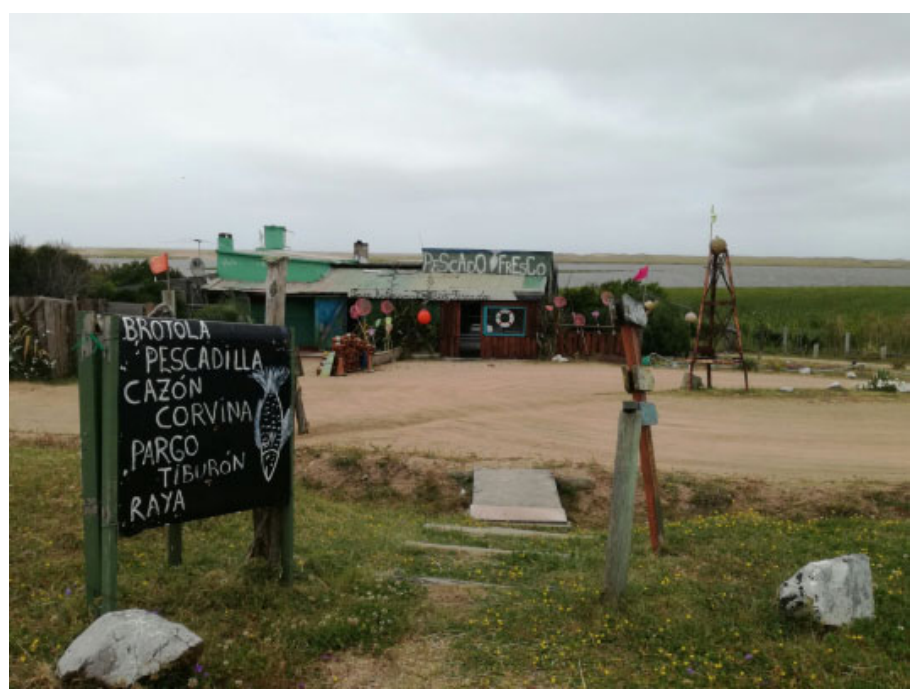

Fuente: Inti Clavijo.

\section{APERTURA DE LA BARRA}

En el caso de estudio presentado aquí, la "apertura de la barra" surge también como un tema de gran relevancia para los pescadores artesanales. En particular, la preocupación se manifiesta con la apertura artificial mediante máquinas y a destiempo de la barra que comunica la laguna con el mar, lo que ha generado un conflicto socio-ambiental que pone en jaque la continuidad de la pesquería. Pues ha sido una de las causas principales, desde la observación de los pescadores, de la ausencia de peces.

De acuerdo a lo que observamos en el trabajo de campo en la actualidad, no hay consenso para su apertura, es decir, cuándo debe hacerse este procedimiento y bajo qué parámetros. Cabe destacar que, en otras lagunas como la de Rocha existe un sistema de manejo que entre otras cosas contempla un protocolo que fija las pautas para la apertura de la barra. Tal como señalan Lagos et al:

"El sistema socio-institucional de Laguna de Rocha, tiene como base un sistema de manejo bien definido a través de la figura de área protegida con marco regulatorio delimitado tanto por el plan de manejo, como por las normativas que rigen a la pesca artesanal y los acuerdos consuetudinarios de la comunidad" (LAGOS et al 2017, 12).

Es importante señalar que dicho protocolo se realizó contemplando e incluyendo el conocimiento generado en las prácticas de la localidad por pescadores artesanales que antiguamente realizaban la apertura manual de la barra, cuando la misma estaba a punto de caer.

De acuerdo a lo planteado por los pescadores en los últimos años, la barra de Laguna Garzón se abre antes de tiempo y no dura lo suficientemente abierta como para que entren las especies y el agua salada. Algunos señalan que la barra se abre de acuerdo a los intereses de unos pocos afectando a la mayoría. La dificultad más significativa es que, no están claros cuáles son los criterios de apertura y esto genera diferencias y conflictos entre los actores que allí conviven y los que gestionan el proceso de apertura artificial con máquina. 
En tal sentido, un interlocutor nos comentaba que actualmente la barra se abre menos- refiriéndose a la profundidad- $\mathrm{y}$ como no está muy honda la laguna entra menos pescado:

\begin{abstract}
"Et: (...) y uno lo compara también con la laguna de José Ignacio y un poco te das cuenta que, que puede ser por eso, por tanta tierra que dejaron ahí porque allá entra pescado, y acá se abre mucho menos y ta', cuando está bajo entra mucho menos pescado" (Entrevista Pescador artesanal, mayo 2018).
\end{abstract}

Otro pescador nos describía que, la barra la abren a máquina, “... viste cuando se inunda mucho, la abren a máquina y después se cierra" (20 de abril de 2018). En este sentido, el tema prioritario para la gestión sería definir el nivel preciso en el cual es conveniente proceder a su apertura. Este mismo pescador nos relató que, anteriormente la barra se abría manualmente, se ponían de acuerdo los pescadores y entre todos iban y abrían la barra con palas:

\begin{abstract}
"Et:... por eso te digo nosotros pasábamos una semana, nosotros íbamos de acá a pie a la barra, de acá a pie a la barra, con una pala al hombro, tres días, tres días a excavar la barra veníamos de tardecita sin comer y sin nada pa' las casas, dormíamos al otro día tomábamos mate y vuelta a la barra, pa' poderla abrir.
\end{abstract}

Er: ¿Iban todos los...?

Et: En ese momento había ocho, diez pescadores iban todos, pero ya sabíamos que estaba para abrirla ahora que hay buen tiempo que hay corvina negra, o que hay lisa en la costa o que hay pejerrey y después que sabemos que va a durar un mes abierto, no tres días, la última vez duró tres días abierta, nunca en mi vida lo había visto yo" (Entrevista Pescador artesanal, noviembre 2017).

La apertura de la barra artificial con máquinas viene siendo presentada públicamente por los pescadores como un problema desde hace varios años; ya en 2011 apareció en la prensa la denuncia del problema. Sin embargo, desde los diversos organismos encargados de la gestión, durante muchos años los temas prioritarios han involucrado otros elementos de la zona como la protección del monte samófilo, el impacto de la creación del puente, el loteo y desarrollo urbano en el área. Aspectos que pareciera reflejar una gestión más enfocada en los problemas "terrestres" presentes en el área que en los "acuáticos". Si bien en el último año, debido a las presiones de diversos actores, entre los que se encuentran pescadores artesanales, ONG'S e investigadores, se iniciaron las tratativas para generar un Convenio entre la Universidad de la República y la DINAMA, y desarrollar un Protocolo de apertura de la barra considerando las diversas perspectivas, como fue realizado en el caso antes mencionado en Laguna de Rocha. 


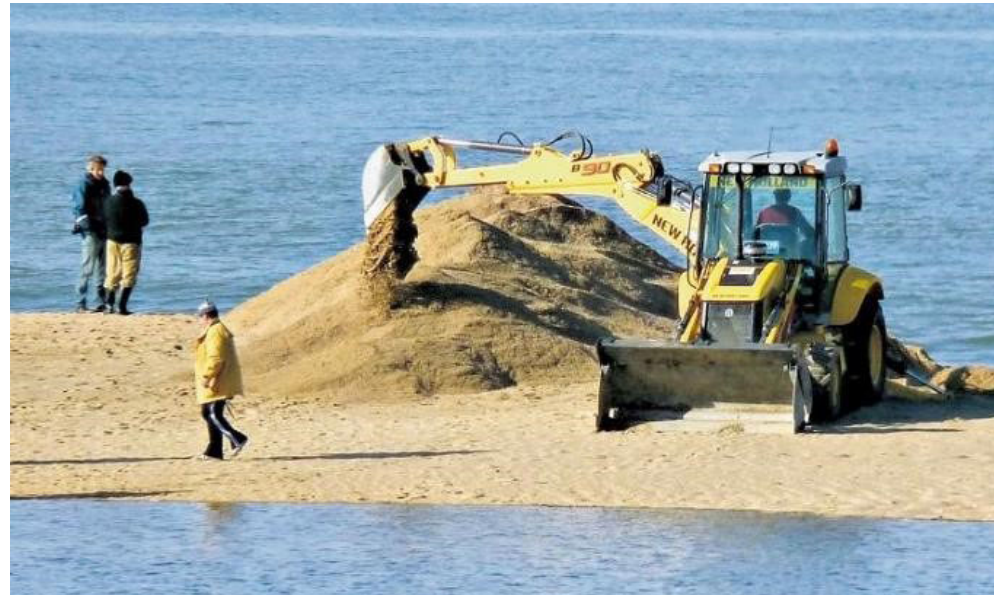

Fuente: foto extraída de prensa, link en notas (viii).

Figura 9 - Apertura de la barra de Laguna Garzón (2007)

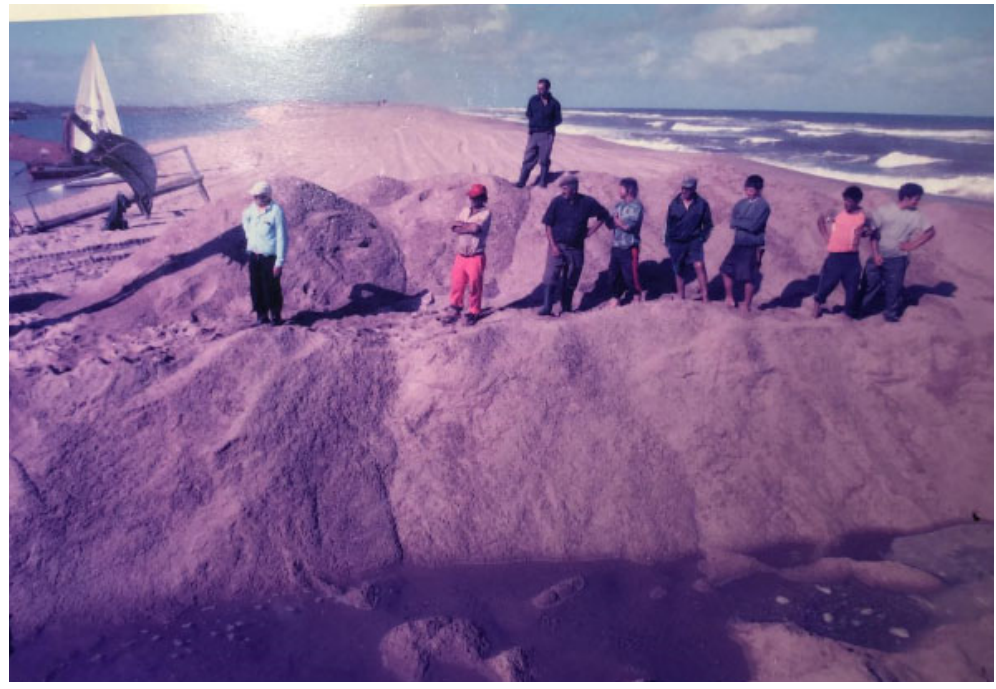

Fuente: Carlos Amorin (Pescador artesanal)

\section{CONCLUSIONES}

A modo de cierre, presentamos algunas conclusiones que refieren a distintos niveles de análisis, en primer lugar nos referiremos a aspectos que dan cuenta de la particularidad y al caso concreto de estudio, y por otro, a reflexiones que forman parte del trabajo de campo y la investigación con colectivos dedicados a las pesquerías artesanales.

Con respecto a las territorialidades podemos decir que, los pescadores artesanales entienden el espacio de Laguna Garzón en relación con otras lagunas, puesto que existe un tipo de movilidad entre lagunas vinculadas a las distintas faenas pesqueras y zafras. La movilidad aparece con frecuencia en los relatos de los interlocutores de esta investigación, muchos ya dejaron de moverse y optaron por quedarse asentados en Garzón pescando solamente allí, aunque las capturas mermen considerablemente, más bien la movilidad que observamos en la actualidad tiene que ver con pescadores de otras lagunas que vienen ocasionalmente a faenar a Laguna Garzón y como una referencia a la pesca que realizaban anteriormente. 
Asimismo, lo que observa Diegues para el caso de la territorialidad marina, lo vemos en el caso estudiado de la laguna cuya territorialidad "depende no solamente del medio físico a ser explorado, sino que también de las relaciones sociales establecidas entre aquellos que lo utilizan" (DIEGUES 2001, 6). Como vimos, en Laguna Garzón cuentan con un conocimiento local de geo-referenciación producido por los pescadores, el cual consiste en la delimitación de lugares que funcionan como referencia, en tal sentido, los nombres que se le asignan a dichos lugares se corresponden tanto con las características del agua, con "piedras o cacholetas", o finalmente son los nombres de algunas chacras cercanas. Este sistema les permite rápidamente ubicar y geo-referenciar los lugares de pesca. En relación a lo anterior una temática interesante que surgió en la investigación fue la vinculada con los "secretos" en la pesca que, como vimos, ha sido un tema recurrente en la antropología especializada en la temática pesquera. Aunque exista esa manera de comunicar y ubicar los lugares de pesca, no siempre se comparte el éxito en la faena, como una manera de asegurar $u$ optimizar las capturas. Sin embargo, como pudimos observar, los pescadores desarrollan relaciones morales y afectivas que incluyen acuerdos del orden de la solidaridad en cuanto a competencia tecnológica y participación común en el proceso de trabajo (MALDONADO, 1994).

La Laguna y su entorno, en particular sus aguas, son entendidas por los pescadores como una materialidad de características sumamente mutables y dinámicas, en las que inciden diversas agencias, como vimos anteriormente: vientos, corrientes de agua salada, corrientes de agua dulce que puede ser el agua del arroyo, así como el agua de lluvia; estos elementos son algunos de los que inciden, de acuerdo a los interlocutores de este trabajo, en la posibilidad de encontrar peces. Recientemente vemos que, a estos elementos se le suman otros como la agencia humana y no humana de las máquinas. Esta última es vista como una amenaza para mantener el dinamismo y las características propias del agua de la laguna que garantizan la presencia de peces. Esta entidad dinámica se ve afectada por la apertura a destiempo de la barra de la laguna, de acuerdo a la perspectiva de los pescadores. Así, los intereses de distintos actores sociales se ven desencontrados, generando conflictos que no han llegado a resolverse de forma exitosa hasta el momento. Sin embargo, consideramos que, por la complejidad y genealogía, la cual data de varias décadas de observación y práctica, el conocimiento generado en las prácticas de la localidad de los pescadores residentes desde hace varias décadas en la zona circundante a la laguna, debiera ser considerado en los planes de manejo y en los protocolos de apertura de la laguna que se busca desarrollar.

\section{NOTAS DE FIM}

${ }^{1}$ Trabajo presentado en la $31^{\text {a }}$ Reunión Brasilera de Antropología, realizada entre los días 09 y 12 de diciembre de 2018, Brasilia/DF.

${ }^{2}$ Instrumentos de navegación marítima que utilizan el eco acústico para medir la distancia con el fondo del lecho acuático.

${ }^{3}$ En lo sucesivo Er: significa entrevistador y Et entrevistado. Por razones de confidencialidad se omitieron los nombres de los entrevistados.

4 "Chacra" es una forma de denominar a las fincas de campo de mediana extensión en las zonas rurales uruguayas.

${ }^{5}$ El concepto de panóptico definido por Foucault (2001) de forma muy precisa y completa, en este caso es utilizado para comprender la forma de observación que dispone la arquitectura y el diseño de las casas de la Laguna, dónde: "Este panóptico, sutilmente dispuesto para que un vigilante pueda observar, de una ojeada, a tantos individuos diferentes, permite también a todo el mundo venir a vigilar al vigilante de menor importan- 
cia. La máquina de ver era una especie de cámara oscura donde espiar a los individuos (...)" (Foucault 2001:204). Así, desde las pequeñas ventanas de las casas de los pescadores pueden ver lo que ocurre en la costa de la laguna de forma oculta.

${ }^{6}$ Existen casos similares en otras lagunas costeras del este uruguayo como la experiencia de Laguna de Rocha, para un acercamiento general del caso pueden leerse artículos de prensa como el siguiente: https://ladiaria.com.uy/articulo/2019/5/investigadores-desarrollaron-protocolo-de-apertura-de-la-barra-de-arena-en-laguna-de-rocha/ Por información académica en mayor profundidad se puede acudir al artículo citado de LAGOS, Ximena; DABEZIES, Juan; DELGADO, Estela; CETRULO, Ricardo (2017).

${ }^{7}$ Esto hace referencia a la tierra empleada para el "rescate" de una retroexcavadora que sufrió un accidente y se hundió en la laguna mientras se realizaban las obras del puente circular: http://lapalomahoy.uy/nota/1528/una-retroexcavadora-se-hundio-en-laguna-garzon

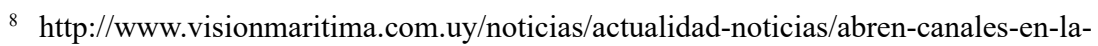
gunas-garzon-y-rocha/

\section{REFERENCIAS}

ADOMILLI, Gianpaolo. Terra e mar, do viver e do trabalhar na pesca marítima: Tempo, espaço e ambiente junto a pescadores de São José do Norte- RS. Tese de Doutorado. Porto Alegre: PPGAS - UFRGS, 2007.

COLAÇO, José. Quanto custa ser pescador artesanal?: Etnografia, relato e comparação entre dois povoados pesqueiros no Brasil e em Portugal. Río de Janeiro: Garamond, 2015.

D'AMBROSIO, Leticia; LEMBO, Victoria; AMATO, Blas; THOMPSON, Diego. El mundo sumergido: Una investigación antropológica de la pesquería del mejillón en Piriápolis y Punta del Este. Montevideo: Facultad de Humanidades y Ciencias de la Educación, 2010.

D’AMBROSIO, Leticia. La experimentación perceptual de la costa y el mar: Un estudio con surfistas, biólogos y pescadores artesanales. Brasil: Revista Tessituras Furg, 2017a.

D’AMBROSIO, Leticia. Leer el mar: habitando la costa, la tierra y el mar con surfistas, pescadores artesanales y biólogos, en la Costa Este de Uruguay. Tesis de Doctorado en Antropología Social. Argentina: IDAES-UNSAM, $2017 \mathrm{~b}$.

DIEGUES, Antonio. Saberes tradicionais e biodiversidade no Brasil. Ministério do Meio Ambiente - MMA, 2001.

FOUCAULT, Michel. Vigilar y castigar: nacimiento de la prisión. 1A Ed. Buenos Aires. Siglo veintiuno editores, 2001.

GALVÁN TUDELA, Alberto y FERNÁNDEZ, Pascual (1996). Pescadores: la sociedad de pescadores y la antropología. En: Prat, J. y Martines, A. (eds.) (1996), Ensayos de antropología social: homenaje a Claudio Esteva Fabregat. Barcelona. Disponible en: http://www.antropologiasocial.org/contenidos/publicaciones/Jpascual/Pescado- res.pdf. Acceso en: oct. 2012.

HOBSBAWM, Eric; RANGER, Terence (eds.) La invención de la tradición. Barcelona: Ed. Crítica, 2002.

INGOLD, Tim; KURTILA, Terhi. Perceiving the Environment in Finnish Lapland. Body \& Society, v. 6, págs. 183-196. 2000.

LAGOS, Ximena; DABEZIES, Juan; DELGADO, Estela; CETRULO, Ricardo. Vínculos para la gestión: dinámicas socioinstitucionales y perspectivas para el manejo integrado de la pesca artesanal en Laguna de Rocha (Uruguay). REDES Revista Hispana para el Análisis de Redes Sociales Vol. 28,\#1, 47-60, 2017. 
MALDONADO, Simone. Mestres e mares: espaço e indivisão na pesca marítima. San Pablo: Annablume Editora, 1994.

MOURA, Gustavo; DIEGUES, Antônio. Os conhecimentos tradicional e científico do saco do Arraial, Estuário da Lagoa dos Patos (RS), B. Inst. Pesca, São Paulo, 35 (3): 359-372. (2009).

TEIXEIRA DE MELLO, F; GONZÁLEZ-BERGONZONI, I. \& LOUREIRO, M. (2011). Peces de agua dulce del Uruguay. PPR-MGAP. 188 pp. Disponible en: http://www.mgap.gub.uy/sites/default/files/multimedia/10_peces_de_uruguay_2011.pdf.

SIMMEL, Jorge. Estudios sobre las formas de socialización V: el secreto y la sociedad secreta. Revista de Occidente, tomo III, Madrid. 1927. 